\title{
Investigation of coercivity mechanism in hot deformed Nd-Fe-B permanent magnets by small-angle neutron scattering
}

\author{
M. Yano, ${ }^{1, a)}$ K. Ono, ${ }^{2}$ M. Harada, ${ }^{3}$ A. Manabe, ${ }^{1}$ T. Shoji, ${ }^{1}$ A. Kato, ${ }^{1}$ and J. Kohlbrecher ${ }^{4}$ \\ ${ }^{1}$ Advanced Material Engineering Division, Toyota Motor Corporation, Susono 410-1193, Japan \\ ${ }^{2}$ High Energy Accelerator Research Organization (KEK), Tsukuba 305-0801, Japan \\ ${ }^{3}$ Toyota Central R\&D Labs, Inc., Aichi 480-1192, Japan \\ ${ }^{4}$ Laboratory for Neutron Scattering, Paul Scherrer Institut, 5232 Villigen PSI, Switzerland
}

(Presented 7 November 2013; received 22 September 2013; accepted 22 November 2013; published online 24 February 2014)

The magnetic reversal behaviors of single domain sized Nd-Fe-B permanent magnets, with and without isolation between the $\mathrm{Nd}_{2} \mathrm{Fe}_{14} \mathrm{~B}$ grains, was clarified using small-angle neutron scattering (SANS). The SANS patterns obtained arose from changes in the magnetic domains and were analyzed using the Teubner-Stray model, a phenomenological correlation length model, to quantify the periodicity and morphology of the magnetic domains. The results indicated that the magnetic reversal evolved with the magnetic domains that had similar sized grains. The grain isolation enabled us to realize the reversals of single domains. (C) 2014 AIP Publishing LLC. [http://dx.doi.org/10.1063/1.4866841]

\section{INTRODUCTION}

The high maximum energy product of Nd-Fe-B magnets enabled the fabrication of efficient electric motors or generators. ${ }^{1} \mathrm{Nd}-\mathrm{Fe}-\mathrm{B}$ magnets in hybrid and electric vehicles require high coercivity in order to maintain their magnetization, even at operating temperatures up to $\sim 20{ }^{\circ} \mathrm{C}$. To obtain high coercivity Nd-Fe-B magnets, Dy is an indispensable element used to enhance the magnetic anisotropy fields. ${ }^{2}$ However, Dy is a rare-earth element. Moreover, Dy reduces the magnetization in Nd-Dy-Fe-B magnets because of the antiparallel coupling between Dy and Fe. ${ }^{3,4}$ Therefore, enhancement of the coercivity without Dy is important in developing next generation Nd-Fe-B magnets.

One of the possibilities to enhance the coercivity of NdFe-B magnets is reducing the size of grains. Although this is known in the past, only the grain size refinement may not lead to higher coercivity. From recent works, it was found that the isolation between grains is important for enhancement of the coercivity. When the isolation is not enough, neighboring grains magnetically interact with each other by exchange interaction then the reversed domains connect and behave like a larger reversal unit during the reversal procedure. ${ }^{5}$

In this work, to clarify the effect of grain isolation in single domain sized Nd-Fe-B magnets, the reversal event was observed using small-angle neutron scattering (SANS), which is a bulk magnetic domain observation technique.

\section{EXPERIMENTAL}

The nano-crystalline hot-deformed Nd-Fe-B magnets were made from rapidly quenched melt spun-spun ribbons. The melt-spun ribbons were crushed into powders a few hundred microns in size and then sintered at $873 \mathrm{~K}$ under a

\footnotetext{
a) Author to whom correspondence should be addressed. Electronic mail: masao_yano_aa@mail.toyota.co.jp
}

pressure of $100 \mathrm{MPa}$. This sintered bulk was hot-deformed with a height reduction of about $80 \%$ to develop (001) texture of $\mathrm{Nd}_{2} \mathrm{Fe}_{14} \mathrm{~B}$ phase. The texture degree, ratio of the remanence and saturation magnetization, was about 0.9. To isolate grains, the hot-deformed magnet was soaked in a molten $\mathrm{Nd}-\mathrm{Cu}$ alloy at $873 \mathrm{~K}$ for $120 \mathrm{~min}$ to infiltrate the $\mathrm{Nd}-\mathrm{Cu}$ alloy to melt into the grain boundaries. ${ }^{6}$ Herein, this sample is called the infiltrated sample. The coercivities of these hot-deformed and infiltrated samples were about 1.8 and $2.2 \mathrm{~T}$, respectively.

The SANS experiments were performed at SANS-I at the Paul Scherrer Institut (PSI). ${ }^{7}$ All measurements were performed in a vacuum at room temperature. An electromagnet was used to apply magnetic fields between $\pm 5 \mathrm{~T}$ to the sample. The sample thickness was $0.5 \mathrm{~mm}$ to avoid multiple scattering. Samples were prepared with the magnetization in the direction of the easy axis in the plane of the sample. We applied a magnetic field parallel to the direction of the easy axis in the sample and perpendicular to the incoming neutron beam. A " $\mathrm{q}$ " range, whose unit is the inverse of length, is important for spatial resolution. The range $0.02 \mathrm{~nm}^{-1}<\mathrm{q}<0.2 \mathrm{~nm}^{-1}$ was covered by using a wavelength of $1 \mathrm{~nm}$ and a detector distance of $15 \mathrm{~m}$. All the SANS data were corrected with a standard procedure using the software package BerSANS. ${ }^{8}$ The magnetic properties of the sample were measured using a vibrating sample magnetometer (VSM) at room temperature after the samples had been completely magnetized at $10 \mathrm{~T}$.

\section{RESULTS AND DISCUSSION}

Figs. 1(a) and 1(b) show the magnetization curves and their corresponding magnetic field dependent SANS intensities for the hot-deformed and infiltrated samples, respectively. In each SANS image, the magnetic field was applied along the horizontal direction, parallel to the easy magnetization $c$ axis of these samples. The SANS patterns were elongated horizontally. This elongation arose from nuclear 


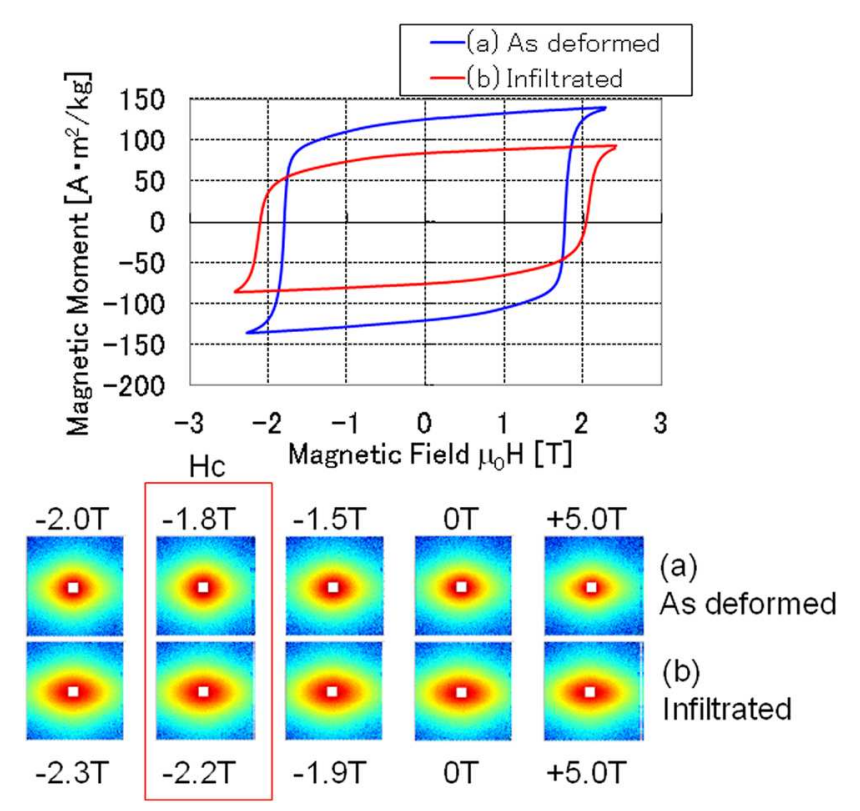

FIG. 1. (Upper) Hysteresis loops of the as-deformed and infiltrated magnets. (Lower) SANS intensities for both samples at different magnetic fields with a $q$ in the range of 0.02 to $0.2 \mathrm{~nm}^{-1}$. The magnetic field was applied along the horizontal direction, which was parallel to the $\mathrm{c}$ axis of the sample. The (a) as-deformed and (b) infiltrated samples.

scattering in the $\mathrm{Nd}_{2} \mathrm{Fe}_{14} \mathrm{~B}$ grains in the hot-deformed nano-crystalline magnets. It is well known that the hot-deformed $\mathrm{Nd}-\mathrm{Fe}-\mathrm{B}$ magnet had platelet shaped $\mathrm{Nd}_{2} \mathrm{Fe}_{14} \mathrm{~B}$ grain and, from the neutron incident direction, grain shape appeared to have a rectangular elongation perpendicular to the $c$ axis. The horizontally longer SANS patterns, after infiltration, implied that grain isolation occurred along the horizontal direction, which is comparable to the previous transmission electron microscopy (TEM) observations. ${ }^{6}$

The SANS pattern in Fig. 1 consists of contributions from both nuclear and magnetic scattering. ${ }^{9}$ Separation of the magnetic and nuclear contributions was realized by subtracting the SANS pattern of the magnetically saturated state. We used the saturated state pattern at $+5 \mathrm{~T}$ from the SANS pattern of each magnetic field state and assumed that the nuclear scattering was field independent. Using this procedure, we were able to extract the magnetic contribution.

To analyze the field dependent magnetic scattering, the q-dependent intensity was integrated along the vertical direction (Fig. 2), because the magnetic scattering is a function of $\sin (2 \alpha)$, where $\alpha$ is the angle from the direction of the magnetic field (horizontal) in the SANS images. The intensity increase near the coercive field $(\mathrm{Hc})$ is thought to have been caused by the appearance of instantaneous domain reversals, related to the magnetization curve in Fig. 1.

The q-dependency of the magnetic scattering intensity, I(q), was fitted using the phenomenological magnetic correlation length model. The functional form was identical to the Teubner-Strey model, ${ }^{10}$ which is well known for analyzing the structures of microemulsion systems. From the mathematical point of view, the evolution equations of the microemulsion system and micromagnetics have a mathematically analogous structure. We have adopted this model to analyze

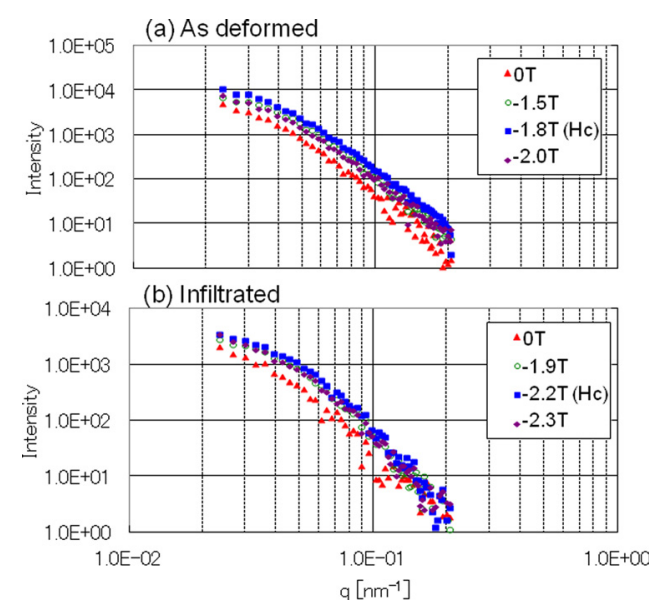

FIG. 2. Integrated intensities I(q) whose intensities were different to the intensities at $+5 \mathrm{~T}$ for the (a) as-deformed and (b) infiltrated samples. The intensity was integrated from the center of $(\mathrm{qx}, \mathrm{qy})=(0,0)$ to the vertical direction in Fig. 1 with a width of $\pm 10^{\circ}$.

the magnetic domain structures. The function in the form of $\mathrm{I}(\mathrm{q})$ is

$$
\mathrm{I}(\mathrm{q}) \approx 1 /\left(\mathrm{a}_{2}+\mathrm{c}_{1} \mathrm{q}^{2}+\mathrm{c}_{2} \mathrm{q}^{4}\right)
$$

where $\mathrm{a}_{2}, \mathrm{c}_{1}$, and $\mathrm{c}_{2}$ are the fitting parameters for this model. Determining these parameters from the SANS patterns gives quantified information on the structures of the domains. From these parameters, the characteristic domain periodicity, $d$, and correlation length, $\xi$, can be calculated using the formulas

$$
\begin{gathered}
d=2 \pi\left[1 / 2\left(\mathrm{a}_{2} / \mathrm{c}_{2}\right)^{1 / 2}-1 / 4\left(\mathrm{c}_{1} / \mathrm{c}_{2}\right)\right]^{-1 / 2}, \\
\xi=\left[1 / 2\left(\mathrm{a}_{2} / \mathrm{c}_{2}\right)^{1 / 2}+1 / 4\left(\mathrm{c}_{1} / \mathrm{c}_{2}\right)\right]^{-1 / 2} .
\end{gathered}
$$

The domain complexity can be described using one of the order parameters $C_{3}$

$$
C_{3}=2 \pi\left[r^{2}-1\right]^{3 / 2} / r^{2},
$$

where $r=2 \pi \xi / d$. Higher $C_{3}$ values, $C_{3} \sim 10$, tended to show droplet like structures, where the domains were separated from each other. Lower $C_{3}$ values had bi-continuous structures, where the domains were connected in a complex structure. ${ }^{11}$ The magnetic field dependencies of $d$ and $C_{3}$ are shown in Fig. 3. When a demagnetizing field was applied, the magnetic field dependency of $d$, estimated using Eq. (2), showed a peak near the Hc. This was caused by dominant magnetic spin direction switching near the Hc. The magnetized direction of the domain was dominant when $|\mathrm{H}|<|\mathrm{Hc}|$ and the reversed domain was dominant when $|\mathrm{H}|>|\mathrm{Hc}|$. At the $\mathrm{Hc}$, the quantity of the positive and negative magnetic spins should be the same. The average periodicity should have a maximum value in this situation because the periodicity reflects the structure of the majority domains. Near the $\mathrm{Hc}$, the value of $d$ was estimated to be $200-250 \mathrm{~nm}$ and $180-230 \mathrm{~nm}$ for as-deformed and infiltrated samples, 

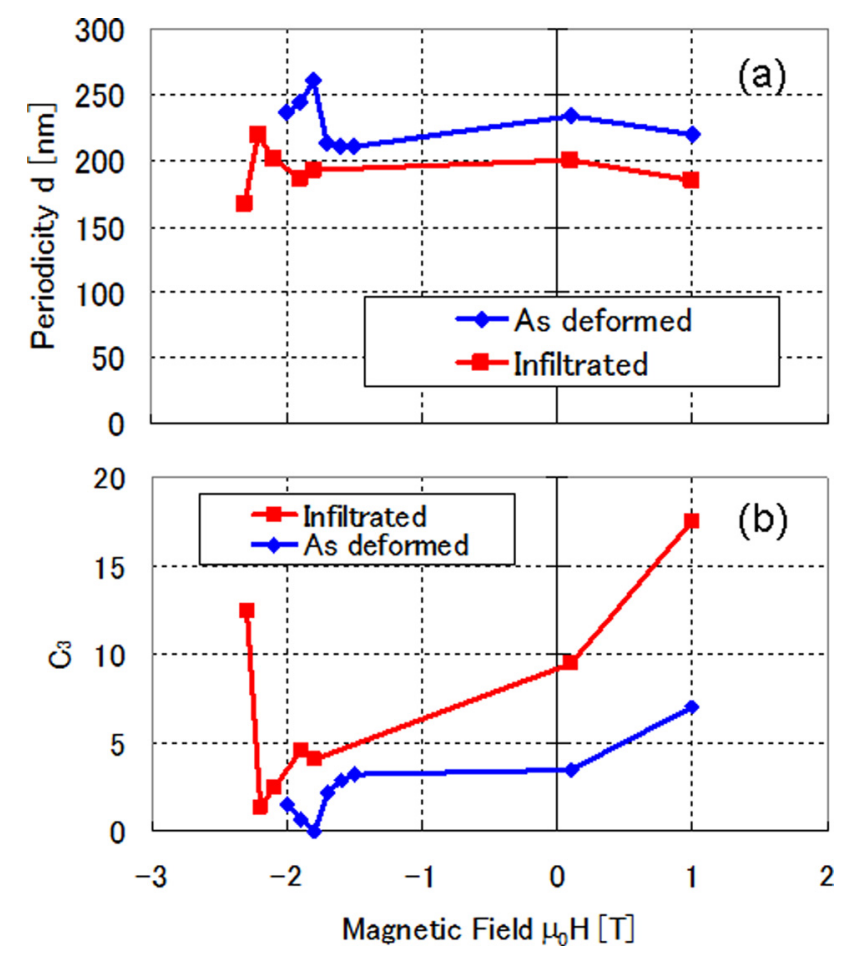

FIG. 3. Magnetic field dependence of (a) the periodicity of magnetic domains and $(b)$ the order parameter $\left(C_{3}\right)$ estimated by fitting the TeubnerStrey model from the graphs in Fig. 2.

respectively. This implied that the domain wall spacing was almost the same size as the size of the domain and was comparable to grain size perpendicular to the easy axis. However, the smaller $d$ value for the infiltrated sample indicated that the domain size was smaller than the as-deformed one. Therefore, that the infiltration caused the domains to become magnetically isolated along the axis perpendicular to the easy axis.

The $C_{3}$ value was largest just after saturation; $C_{3}>10$ for the infiltrated and $C_{3}<10$ for the as-deformed samples. This result indicated that the reversed domains were perfectly isolated just after saturation in the infiltrated sample but were not perfectly isolated in the as-deformed sample. The $C_{3}$ value was the smallest near the Hc, indicating that the magnetic domain structure was bi-continuous in both samples. Even though the domain structure at the Hc was almost the same from in terms of domain complexity, the reversal process was thought to differ. The smaller $C_{3}$ values corresponded to the situation where the reversal domains had a bi-continuous structure, indicating that the domain evolution progresses was not caused by independent reversals of each grain, but by collective reversals of the grains in the asdeformed sample. Higher $C_{3}$ values during the demagnetization process for the infiltrated sample indicated that the reversed domains were isolated. This enabled suppression of the reduction of the magnetization in the demagnetizing fields, causing an increase in the coercivity.

\section{CONCLUSIONS}

SANS measurements in infiltrated hot-deformed nanocrystalline magnets were performed to clarify the magnetic domain evolution during the magnetic reversal process in bulk magnet systems.

From the Teubner-Stray model fitting, the reversal domain sizes in the infiltrated sample were smaller than that in the as-deformed sample and were comparable to the size of the grains. Also, a droplet domain structure was observed in the infiltrated sample, a smaller reversal unit give reason for the higher coercivity.

\section{ACKNOWLEDGMENTS}

This work was performed at the Paul Scherrer Institut under Proposal No. 20110638. Part of this work was performed under the Magnetic Materials for High-Efficient Motors (MagHEM) project. The authors thank Dr. Y. Takada for the sample preparation.

${ }^{1}$ O. Gutfleisch, J. Phys. D: Appl. Phys. 33, R157 (2000).

${ }^{2}$ K. Kobayashi, K. Urushibata, T. Matsushita, and T. Akiya, J. Appl. Phys. 111, 023907 (2012).

${ }^{3}$ J. F. Herbst and W. B. Yelon, J. Appl. Phys. 57, 2343 (1985).

${ }^{4}$ K. Ono, T. Araki, M. Yano, N. Miyamoto, T. Shoji, A. Kato, A. Manabe, H. Nozaki, Y. Kaneko, and J. Raabe, IEEE Trans. Magn. 47, 2672 (2011).

${ }^{5}$ M. Yano, K. Ono, A. Manabe, N. Miyamoto, T. Shoji, A. Kato, Y. Kaneko, M. Harada, H. Nozaki, and J. Kohlbrecher, IEEE Trans. Magn. 48, 2804 (2012)

${ }^{6}$ H. Sepehri-Amin, T. Ohkubo, S. Nagashima, M. Yano, T. Shoji, A. Kato, T. Schrefl, and K. Hono, Acta Mater. 61, 6622 (2013).

${ }^{7}$ V. K. Aswal, B. van den Brandt, P. Hautle, J. Kohlbrecher, J. A. Konter, A. Michels, F. M. Piegsa, J. Stahn, S. Van Petegem, and O. Zimmer, Nucl. Instrum. Methods Phys. Res. A 586, 86 (2008).

${ }^{8}$ U. Keiderling, Appl. Phys. A 74, S1455 (2002).

${ }^{9}$ A. Michels and J. Weissmüller, Rep. Prog. Phys. 71, 066501 (2008).

${ }^{10}$ M. Teubner and R. Strey, J. Chem. Phys. 87, 3195 (1987).

${ }^{11}$ S. H. Chen, S. L. Chang, and R. Strey, Prog. Colloid Polym. Sci. 81, 30 (1990). 